\title{
MOLECULAR DYNAMICS SIMULATIONS OF IONIC COPOLYMERS
}

\author{
MICHAL BANASZAK \\ Macromolecular Physics Laboratory
Institute of Physics, Adam Mickiewicz University
ul. Umultowska $85,61-614$ Poznań, Poland
}

Abstract: Molecular Dynamics (MD) studies of ionic diblock copolymers are reported. The symmetrical diblocks are shown to exhibit a microphase separation transition (MST) similar to that of neutral diblocks. The MST was investigated by measuring thermodynamic, structural and dynamic properties: density, simulation box dimensions, structure factor, anisotropy in structure factor and directional diffusion constants. The slow cooling of the system from a high-temperature disordered phase to low-temperature ordered microphase separated phase was achieved in both temperature controlled ('NVT') MD, and temperature and pressure controlled ('NPT') MD.

\section{N T R O D U C T I O N}

Ionic polymers (polyelectrolytes, ionomers) are of considerable current interest [1,2]. In those systems one ionic species, e.g., anion, is chemically bonded to the polymer chain either to a pendant group or to the backbone chain; the cation (counterion), on the other hand, is not chemically bonded to the chain and free to move if its thermal energy can overcome the electrostatic forces. The polymer chains can bestow a rubbery mechanical response whilst the counterions are more mobile and provide the electrical conductivity. The microarchitecture of those copolymer-like materials is complex, and also controlls the counterion mobility. In this article we present results of molecular dynamics (MD) simulations of symmetric diblock copolymers in which one block is an ionic polymer and one neutral, and both blocks have nearly the same size. The symmetric diblocks offer also a good model system for comparison with neutral (non-ionic) systems since the neutral diblock copolymers are fairly well understood theoretically and have been extensively studied experimentally and in simulations [3-26]. Unlike many previous simulations, which are Monte Carlo (MC) lattice simulations, our study employs a continuum space molecular dynamics method which is efficient at high densities, can easier be implemented to simulate the constant pressure systems, and finally it is better in calculating both the structural and dynamical properties, for example diffusion constant.

It should be noted that while ionic polymer dilute solutions are fairly well understood, this is not the case for ionic polymer melts. We expect, however, a great deal of both excluded volume interaction screening and electrostatic interaction screening [25].

Since our main interest is in universal properties of the ionic polymer, we employ a coarsegrained model. This means that we are going to ignore the valence angle potential and the torsional potential, and keep only the rigid bonds along the chain, short range repulsive interaction and the electrostatic interactions.

In our simulations we use molecular dynamics with Berendsen loose coupling, either only to thermostat ('NVT'), or both to barostat and thermostat ('NPT') in order to explore the 
microphase separation in ionic nearly symmetric diblock copolymers. While neutral diblock copolymers have been given much attention, in simulations theoretical studies and experimental studies, the ionic copolymers enjoyed less attention because of their complexity. By comparing the microphase behaviour of both ionic and neutral (non-ionic) systems we aim at showing both similarities and differences between them. Since the counterion transport properties in microphase-separated ionic copolymers are of great practical interest, we want to probe the relationship between counterion diffusion and microstructure. Finally by gaining insight into the symmetric diblock case, we lay foundations for our future studies, i.e., simulations of asymmetric ionic diblocks, ionic multiblocks or ionic branched copolymers such as NAFION.

Neutral diblock copolymers can self-assemble into a variety of ordered microstructures. This self-assembly or ordering takes place as those copolymers are cooled down from a disordered high temperature phase to low temperature ordered phase. This process is called order-disorder transition (ODT) or microphase separation transition (MST), and is characterized by a temperature which marks the onset of long range order $T_{O D T}$ It is well established experimentally that the microphase (ordered phase) can look like an array of bcc spheres, hexagonally packed cylinders, or spatially ordered layers. These microphases are often referred to as the "classical" microstructures. Close to the ODT the interface between the microdomains is diffused, and interfaces can fill up most of the space. This segregation regime is called weak segregation limit (WSL). By contrast, when the interfaces between microdomains are sharp and highly localized the system is in strong segregation limit (SSL). The diblock copolymer microstructure in the strong segregation regime is largely determined by volumetric considerations. The diblocks with roughly the same volume of both species form layers (lamellae), while diblock with significant (more than about $80 \%$ by volume) excess of one species form ordered spheres (micelles). The diblocks with intermediate volume fractions usually form hexagonally packed cylinders.

Apart from those 'classical' microstructures, there have been discovered 'non-classical' bicontinuous microstructures such as ordered bicontinuous double diamond (OBDD) structure ( $P n \overline{3} m$ space group), gyroid bicontinuous structure ( $I a \overline{3} d$ space group) and hexagonally perforated layers. There is still some controversy about the existence and thermodynamic stability of those 'non-classical' microstructures. The experimental phase diagram of a real diblock copolymer can be found, for example in reference [10],

Theories of neutral diblock copolymers have been successfully advanced over the past 20 years [6, 11, 22 and 23], both in the strong and weak segregation limits. In the weak segregation regime Leibler's mean-field Random Phase Approximation (RPA) approach [6] was particularly useful. This theory predicts a phase diagram in Flory interaction parameter, $\chi$, multiplied by degree of polymerization, $N$, and diblock composition. When one goes beyond the mean-field approximation, and fluctuations are taken into account in the one-loop approximation [11], then the phase is more complex, but the RPA approach is still very useful in qualitative description of diblock copolymers. In the strong segregation limit there have been many selfconsistent numerical calculations initiated by Helfand and coworkers [23], and also almost analytical calculation presented first by Semenov [22]. 
For ionic copolymer system, the theoretical work done by Marko and Rabin [8] is particularly relevant for our study. They looked at microphase-separation properties of diblock copolymers composed of a neutral polymers joined to an ionic block. For a symmetric diblock, they predict a broad weak segregation region due to a heavy entropic penalty for counterions localization in strongly segregated micro domains. The work by Nyrkova et al. [3] is also interesting because it predicts a new limit for microphase separated ionic copolymers, the super strong segregation limit (SSSL). Their theory is applicable to highly asymmetric multiblock copolymers which form micelles. The chains in those micelles become fully extented in this limit.

Most of the computer simulations of diblock copolymers are lattice simulations. The first three-dimensional Monte Carlo (MC) lattice simulations of block copolymer melts have been performed by Binder and Fried for both symmetric [13] and asymmetric [12] case. These simulations have been extensive yielding numerous significant results and ideas. Other relevant MC studies include the work by Mattice and coworkers [19], Weyersberg and Vilgis [14], and Freire and coworkers [15]. The current MC studies of diblocks, however, are limited by being performed on a lattice. Moreover the relation between MC "time" and physical time is open to debate. We therefore think that off-lattice MD simulations give both better insight and better representation of physical reality.

The recent study of Grest et al. [16] reports an MD off-lattice simulation of a non-ionic diblock copolymer. This study is also interesting because it shows the advantage of simulating under constant pressure. This advantage is the freedom of the system to change the shape and size of the simulation box, and thus to achieve its equilibrium periodicity and microstructure.

The ionic conductivity can in principle be determined directly by MD or NEMD but accurate measurements are difficult. We have instead assumed that the proportionality between conductivity and self-diffusion is a reasonable assumption for our systems. Consequently we have concentrated attention on measurements of cation diffusion coefficients which are expressed as the reduced mean square displacements, without dividing them by the corresponding time.

The reduced density, $\rho^{*}=\sigma^{3} \rho$ (where a is the diameter of the bead), is initially set to 0.5 which is closer to the semidilute regime than to a melt. This low bead density was chosen in order to speed up the equilibration. Our model system consists of twenty 31-mer diblocks and 160 cations, total 780 atoms, confined in a cubic box yielding the desired density. However, some simulations were carried out using 160 chains and 1280 cations. It was necessary to restrict the chain lengths due to very short structural relaxation times in these systems. Although our samples are not in the entangled regime, chains are sufficiently long to differentiate between the chain motions and cation motions.

Finally it should be noted that some of the results presented in this paper has been already reported elsewhere $[25,26]$,

\section{MODEL}

The basic model we apply is a linear freely joined Kramers chain of bead monomers, each corresponding to the size scale of the persistence length of a real polymer. Pairs of neighboring 
beads are connected by links of identical length. Our diblock copolymer, shown schematically in Fig. 1, consists of a neutral block of 15 beads, and the ionic block of 16 beads; in the latter block every second bead carries a negative elementary charge. Eight positively charged beads, i.e., cations, are included to maintain electroneutrality. Volumetricly this makes the system slightly asymmetric in favor of ionic block.

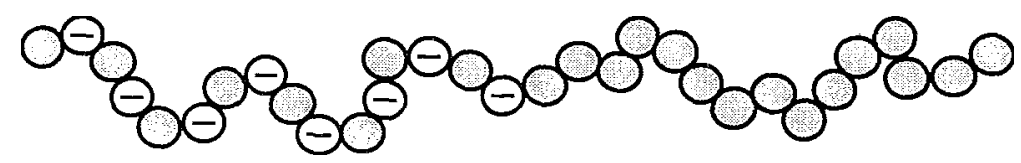

Fig. 1. Schematic picture of the ionic copolymer chain.

We have used a simplified interaction potential which incorporates explicit coulomb contributions superimposed on universal excluded volume interactions. Long range ion interactions are calculated by the Ewald method. The excluded volume effect is modelled using the purely repulsive "WCA" modification of the Lennard-Jones (LJ) potential between interactions centres representing neutral monomers, anionic monomers and "free" cations:

$$
\begin{gathered}
\phi_{W C A}\left(r_{i j}\right)=4 \epsilon\left(\left(\sigma / r_{i j}\right)^{12}-\left(\sigma / r_{i j}\right)^{6}\right)+\epsilon \text { for } r_{i j} \leq 2^{1 / 6} \sigma \\
\phi_{W C A}\left(r_{i j}\right)=0 \quad \text { for } r_{i j}>2^{1 / 6} \sigma
\end{gathered}
$$

Here $\mathrm{r}_{\mathrm{ij}}$ is the separation of monomers $i$ and $j$, and $\sigma$ are the usual LJ parameters which have the same magnitude for all beads. The link length between beads was set equal to $\sigma$.

Including coulomb interactions between the charges the total interaction potential can therefore be written as follows:

$$
\varphi_{\text {TOTAL }}\left(r_{i j}\right)=\left(\frac{q_{i} q_{j} e^{2}}{4 \pi \varepsilon_{o} e_{R} \sigma}\right)\left(\frac{\sigma}{r_{i j}}\right)+\varphi_{W C A}\left(r_{i j}\right)
$$

where the $q$ 's are charge numbers and $e_{\mathrm{R}}$ is the dielectric constant. We can write this a more useful form;

$$
\varphi_{\text {TOTAL }}\left(r_{i j}\right)=E_{\sigma}^{i j} \frac{\sigma}{r_{i j}}+\varphi_{W C A}\left(r_{i j}\right)
$$

where $\mathrm{E}_{\sigma}^{\mathrm{ij}}$ is the electrostatic potential at an ion separation of $\sigma$. Since the unlike charge interaction provides the only cohesive energy in the model we define $\mathrm{E}_{\sigma}$ as a measure of the depth of this potential where $\mathrm{E}_{\sigma}=\left|\mathrm{E}_{\sigma}^{ \pm}\right|$.

The actual location of the minimum of the potential energy between unlike charged beads depends on the ratio $\left(\mathrm{E}_{\mathrm{o}} / \varepsilon\right)$, from now on referred to as $\gamma$. By simple analytical calculations it can be shown that if $\gamma$ is chosen to be 24 , then this minimum is located at $\sigma$, and the depth of the minimum is $((\gamma-1) / \gamma) \mathrm{E}_{\sigma}$ which differs only by about $2.5 \%$ from $\mathrm{E}_{\sigma}$.

For a given value of $\sigma$ temperature is thus reported as $\mathrm{T}^{*}=\mathrm{kT} / \mathrm{E}_{\sigma}$, density as $\mathrm{p}^{*}=\mathrm{N \sigma}^{3} / \mathrm{V}$, pressure as $\mathrm{P}^{*}=\mathrm{P \sigma}^{3} / \mathrm{E}_{\sigma}$, and time as $t^{*}=t\left(\mathrm{E}_{\mathrm{o}} /\left(\mathrm{m \sigma}^{2}\right)\right)^{1 / 2}$, where $m$ is the mass of the bead. The optimized time step $\Delta \mathrm{t}^{*}$ was found to be 0.044 . We should note that the significance of the 
reduced temperature for instance is rather different than in the ordinary LJ system due to extensive mutual screening of the ion interactions in the melt.

In our simulations we measured those properties which are the most useful in determining the ODT and the characterization of cation transport properties. First of all we measured the partial structure factors, defined as follows:

$$
S_{\alpha \alpha}(\vec{k})=\frac{1}{N}\left\langle\left.\left\{\left[\sum_{i=1}^{N_{\alpha}}\left(\sin \vec{k}_{i} \vec{r}_{i}\right)\right]^{2}+\left[\sum_{i=1}^{N_{\alpha}}\left(\cos \vec{k}_{i} \vec{r}_{i}\right)\right]^{2}\right\}\right|_{\text {thermal average }}\right.
$$

where $N$ is the total number of atoms, and is the number of atoms of type $\alpha$, for example cations, and the summation over label $i$ runs through all the atoms of type $\alpha$. In disordered isotropic phase the structure factor can be spherically averaged since all spatial directions are equivalent.

$$
S_{\alpha \alpha}(k)=\left\langle S_{\alpha \alpha}(\vec{k})\right\rangle_{\text {average over all directions }}
$$

However, as the ODT is approached we expect the spherical symmetry to be broken, and accompanied by the rapid growth of the peak in the structure at a characteristic wavelength, $k^{*}$; $2 \pi / k^{*}$ indicates the periodicity of the self-assembling lamellar microphase.

In this study, we show the reduced mean square displacement of cations $\left\langle r_{a}^{2}\right\rangle / \sigma^{2}, \alpha=x, y$, or $z$, as the measure of directional diffusion. We also measured the normalized square end-to-end distance of polymer chain, $\left\langle R^{2}\right\rangle / N \sigma^{2}$, since it increases substantially in the course of ODT. Similarly we report the normalized chain end-to-distance of the ionic block, $\left\langle R_{l}^{2}\right\rangle / N_{1} \sigma^{2}$ and the neutral block, $\left\langle R_{2}^{2}\right\rangle / N_{2} \sigma^{2}$.

\section{RESULTS}

First we report 'NVT' results. In Fig. 2 we show the temperature dependence of four properties which clearly indicate the onset of microphase separation in the ionic copolymer. These results were obtained by cooling an $\mathrm{N}=20$ sample initially prepared as an isotropic disordered melt and equilibrated for $10^{5}$ steps at $\mathrm{T}^{*}=0.096$ which is more than twice the temperature of the ODT. The cooling to $\mathrm{T}^{*}=0.024$ was accomplished incrementally in 14 stages; at each stage the system was equilibrated for $5 \times 10^{4}$ time steps followed by a sampling period of $5 \times 10^{4}$ steps.

At temperatures between 0.096 and $\sim 0.04$ the melt samples were homogeneous and the appearance of a strong nearest neighbour peak in the unlike charge radial distribution functions was an evidence for the existence of counterion condensation on the ionic blocks. Visualization of sample structures revealed that below $\mathrm{T}^{*} \sim 0.04$ the structure became an isotropic and a lamella morphology could be distinguished (see Fig. 3) as would be predicted for a near symmetric block copolymer. In periodic systems the sample spontaneously adopts a layer structure which is commensurate with the periodic boundaries of the simulation cell. The negative and positive ion partial structure factors are indistinguishable for the ordered microphase and in our simulations the peaks in these structure factors show that the lamella periodicity for the $N=20$ system is $\mathrm{L} / 2$ 
( 11.6 a ) and the symmetry of the system is broken in the 100 direction whilst for the $N=160$ sample the periodicity is $L / \sqrt{2}(16.4 \sigma)$, the broken symmetry direction being perpendicular to the 110 direction of the cubic cell. Due to condensed counterions the volume composition of ionic blocks is 0.62 and not 0.5 and this slight imbalance may be the cause of the perforations in the

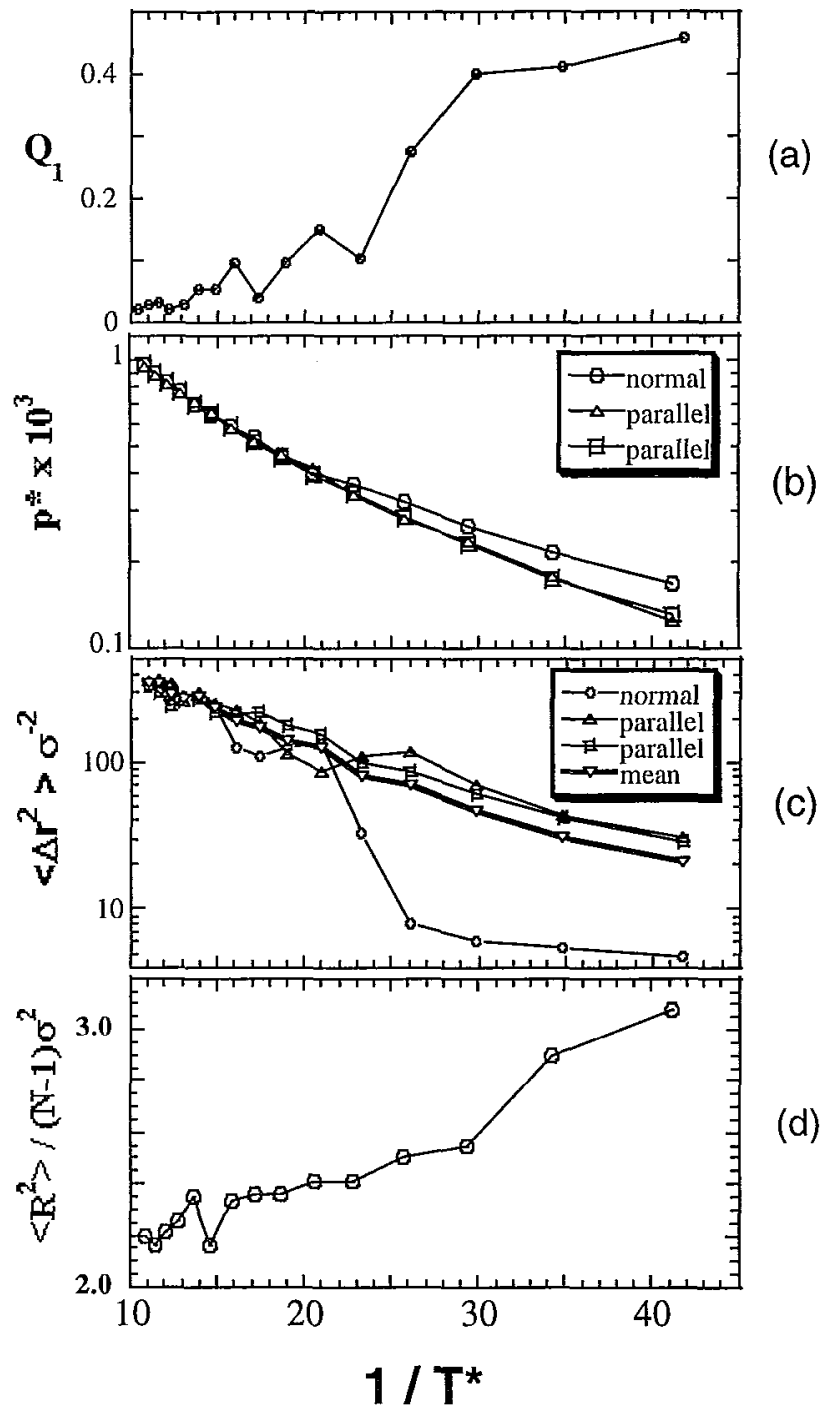

Fig. 2. Inverse temperature dependence of properties showing the onset of microphase separation on cooling the smaller $(\mathrm{N}=20)$ sample: (a) the anisotropy invariant $\mathrm{Q}_{1}$ of the directional structure factor $\mathrm{S}(\mathbf{k})$, evaluated for a spherical shell with $8>\mathrm{kL} / \pi>2$, (b) pressure components normal and tangential to eventual lamella plane of the microphase, (c) mean squared displacements for counterions normal and tangential to eventual lamella plane of the microphase after $5 \times 10^{4}$ time steps, (d) chain mean squared end-to-end distance, showing chain expansion through the ODT. 
neutral layers seen in Fig. 3. Although there is experimental evidence for this kind of 'nonclassical' structure in neutral copolymers [19], we cannot be sure at this stage whether it is truly stable or whether it is a consequence of the boundary restrictions.

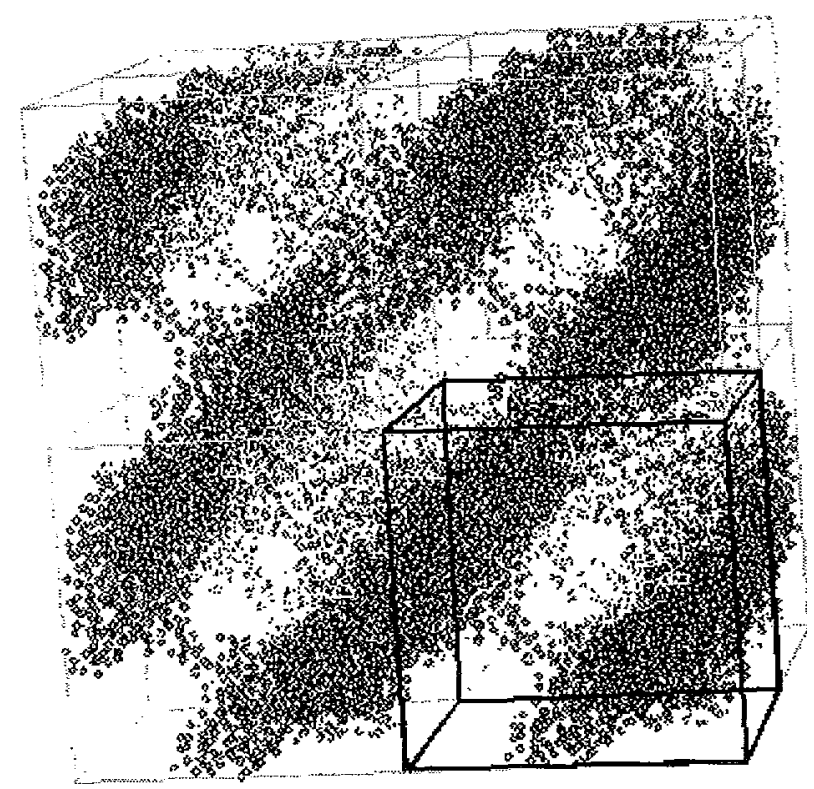

Fig. 3. Instantaneous snapshot of the microphase structure in the 160 chain system at a temperature of $\mathrm{T}^{*}=0.024 ; 8$ neighbouring periodic cells are shown to emphasize the perforated lamella morphology.

The anisotropy of the structure factor [12] shows clearly the onset of structural order for wave vectors corresponding to the low-k peak in the structure factor. The principal moments of the angular distribution of structure factor, $\mathrm{S}(\mathbf{k})$, are ordered such that $\lambda_{1}, \leq \leq \lambda_{3}$. Symmetry breakdown accompanying ODT can then be characterized by two invariants, $Q_{1}$ and $Q_{2}$.

$$
Q_{1}=\frac{\lambda_{2}+\lambda_{3}}{\lambda_{1}}-2
$$

and for lamella symmetry is predicted to be significantly different from zero. It is shown in Fig. $2 \mathrm{a}$ that as the sample is cooled gradually increases and starts to fluctuate. It then changes rapidly to a value of about 0.5 , suggesting that the ODT is at about $\mathrm{T}^{*}=0.043$, while

$$
Q_{2}=\frac{\lambda_{3}+\lambda_{2}}{\lambda_{1}}
$$

is close to zero, as expected.

At high temperature in the disordered phase the diagonal components of the pressure tensor, $P_{x x}, P_{y y}$ and $P_{z z}$, are all equal within the statistical error (Fig 2a), but at about $\mathrm{T}^{*}=0.048$, the pressure in the direction of symmetry breakdown starts to increase due to the onset of lamella ordering. At $\mathrm{T}^{*}=0.024$ the sample is in the microphase-separated regime and there is a significant difference in the tensor components normal and parallel to the direction of lamella ordering. 
There is a significant increase of the copolymer square end-to-end distance as the system is cooled through the ODT and the two dissimilar blocks become separated in space, as shown in Fig. 2(d). Similar effects have been noted in simulations of non-ionic copolymers [16]. There are departures from Gaussian ideality even in the initial (high-temperature) regime but this chain expansion is largely due to fact that the system is in the semidilute regime. In contrast to the case of the overall dimensions the ionic blocks show remarkably little extension over the whole temperature range and in the case of the ionic blocks we attribute this to efficient electrostatic screening of charged monomer repulsions by condensed counterions. Screening of this kind have also recently been observed in simulations of isolated ionic chains [5] for coulomb interaction strengths above the critical value for Manning condensation. In that case the collapse was interpreted in terms of dipole interactions between ion pairs [20]. In the ionic copolymer melt the electrostatic screening is even more effective and the ionic structure below the ODT is comparable with that of a molten salt [21],

A quantity of considerable interest in these ionic systems is the counterion diffusion constant which is related to the ionic conductivity. As the system undergoes separation the counterions distribute themselves in the alternate ionic layers and consequently the counterion mobility in a direction normal to the layers decreases rapidly at the ODT as shown in Fig 2c. In the direction parallel to the lamella planes the diffusional mobility remains high over the whole temperature range studied.

The mean squared displacements of the charged monomers were found to be an order of magnitude smaller than the counterions at long times and this decoupling of the diffusional motions is another important consequence of electrostatic screening. The high counterion mobility in the region of the ODT can also be related to the low value of the glass transition temperature for the ionic blocks. In the case of homopolymers with the same composition as the ionic blocks studied here but at much higher density of 0.93 the glass transition appears at $\mathrm{T}^{*} \sim 0.014$ [18]; for the lower density used here one expects the glass transition to be at an even lower temperature.

Now we turn our attention to 'NPT' results. These results were obtained by cooling an $\mathrm{N}=$ 20 sample initially prepared as an isotropic disordered melt at high temperature. The cooling to $\mathrm{T}^{*}$ - 0.024 was accomplished incrementally in 14 stages; at each stage the system equilibration was followed by a sampling period. As we cooled the system, we monitored its properties. In particular, we looked at the square end-to-end distance of the polymer chains, which is expected to increase as we lower the temperature with more dramatic expansion in the vicinity of ODT. We also measured the square end-to-end distances for both blocks, neutral and charged. The results indicated an increase of copolymer square end-to-end distance as the system is cooled [26].

We also measured box dimensions and density, Figure 4 ( $a$ and b). At high-temperature disordered phase all diagonal components, $L_{x}, L_{y}, \mathrm{~L}_{z}$ are equal within the statistical error, but at about $\mathrm{T}^{*}=0.07$, which is close to the ODT, the dimension in the direction of symmetry breakdown starts to increase, suggesting a force driving the ODT. At $\mathrm{T}^{*}=0.024$, which con es- 
Fig. 4. Temperature dependence of (a) density

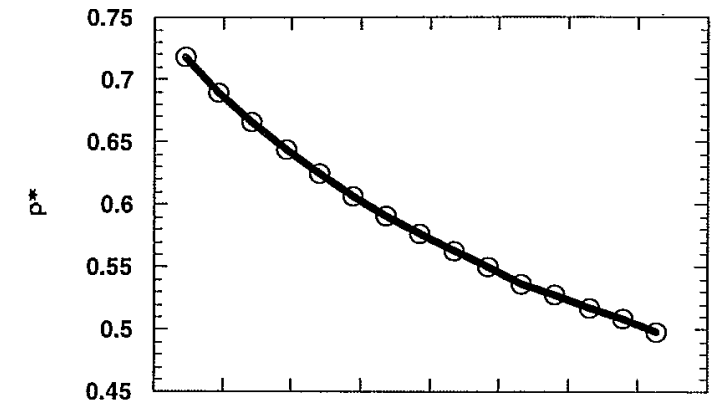

and (b) box dimensions in the 'NPT' cooling process.
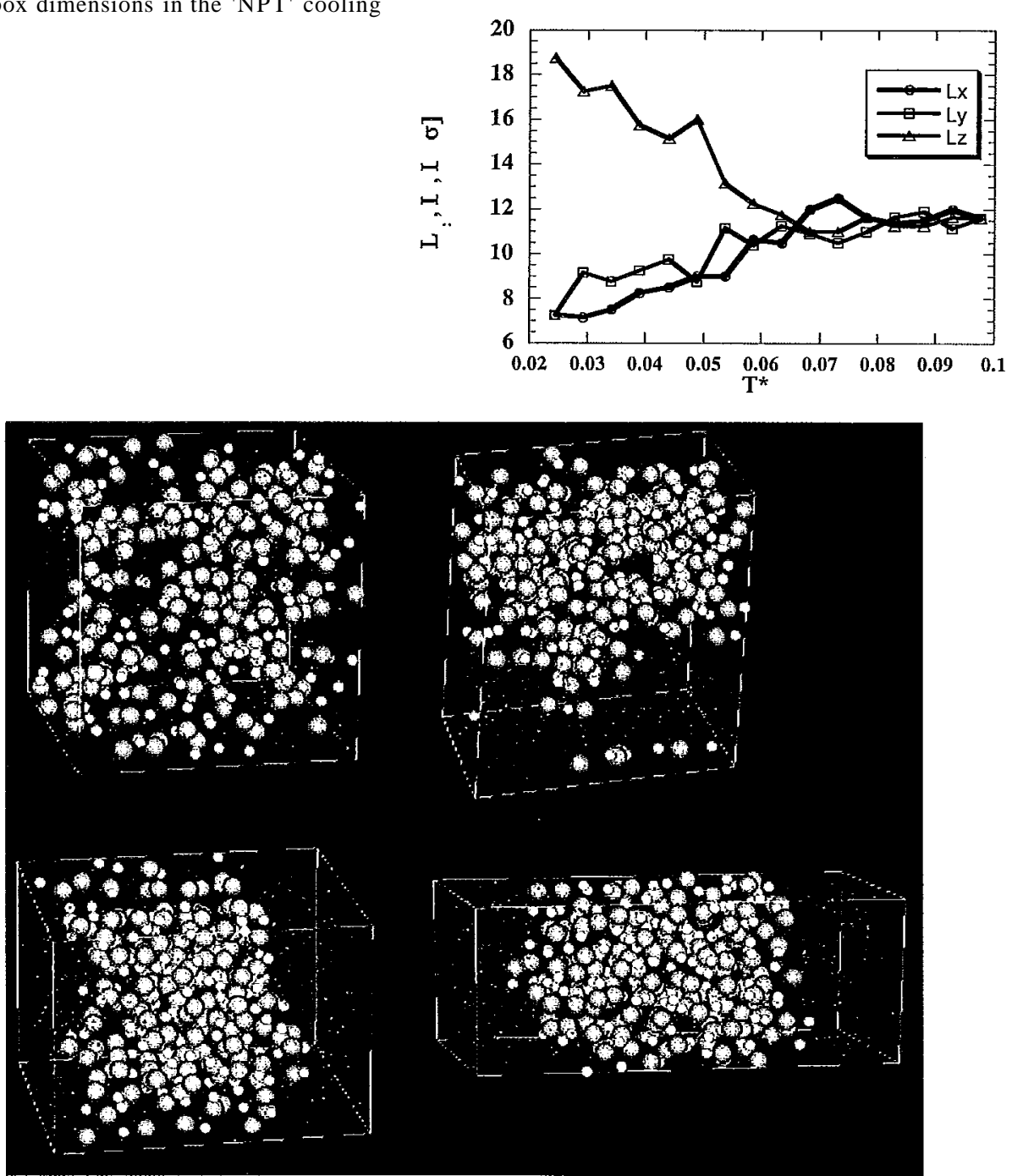

Fig. 5. Snapshots from the 'NPT' simulation (from upper left to lower right): $\mathrm{T}^{*}=0.098,0.073,0.054$ and 0.034 . 
ponds yields a well microphase-separated sample, there is a significant difference in box dimensions. Similarly the density increases as the system is cooled. Snapshots from the simulations (shown in Figure 5) visualize the changes of the simulation box dimensions as the system is cooled.

\section{CONCLUSIONS}

We have presented a generic mesoscopic model for simulation studies which can be used effectively to study microphase separation in ionic copolymers. Ion condensation screens the intensity and range of the electrostatic interactions and for the near symmetric block copolymer studied here this leads to microphase separation which has very similar characteristics to that observed in purely neutral copolymers. It also results in decoupled diffusion of counterions. The dimensions of the microphase in the 'NVT' cooling are distorted by boundary conditions but such effects were reduced by using controlled pressure 'NPT' cooling.

\section{Acknowledgement}

This research was supported by the Polish Committee for Scientific Research (grant no. KBN 8T11F 01214).

\section{References}

[1] Eisenberg, A.; King,M. Ion-Containing Polymers; Academic Press New York

[2] 1977Gouin, J-P.; Bosse, F.; Nguyen, D.; Williams, C. E.; Eisenberg, A., Macromolecules, 26, 7250 (1993)

[3] Nyrkova, I. A.; Khokhlov, A. R.; Doi M., Macromolecules, 26, 3601(1993)

[4] Semenov, A. N.; Nyrkova, I. A.; Khokhlov, A. R., in Ionomers: Characterization, Theory and Applications, Ed. S. Schlick, CRC Press, 1996

[5] Winkler, R. G.; Gold, M; Reineker, P.; Phys. Rev. Lett. 80, 3731(1998)

[6] Leibler, L., Macromolecules, 13, 1602(1980)

[7] Gonzales-Mozuelos, P.; Olvera de la Cruz, M.; J.Chem.Phys. 100, 507 (1994)

[8] Marko, J. F; Rabin, Y.; Macromolecules, 25, 1503(1992)

[9] Bates, F. S.; Fredrickson, G. H.; Ann. Rev. Phys. Chem., 41, 525(1990)

[10] Khandpur, A. K.; Forster, S.; Bates, F. S.; Hamley, I. W.; Ryan, A. J.; Bras, W.; Almdal, K.; Moitensen, K., Macromolecules, 28, 8796(1995)

[11] Fredrickson, G. H.; Helfand, E; J. Chem. Phys. 87, 697(1987)

[12] Fried, H.; Binder, K., J. Chem. Phys., 94, 8349(1991)

[13] Binder, K.; Fried, H., Macromolecules, 26, 6878(1993)

[14] Weyersberg, A.; Vilgis, T. A., Phys. Rev. E, 48, 377(1993)

[15] Molina, L. A.; Freire, J. J., Macromolecules, 28, 2705(1995)

[16] Grest, G. S.; Lacasse, M.-D.; Kremer, K.; Gupta, A. M., J. Chem. Phys., 105, 10583(1996)

[17] Groot, R. D.; Madden, T. J; J. Chem. Phys., 108, 8349(1998)

[18] C. Wong and J. H. R. Clarke, submitted for publication

[19] Matsen, M. W.; Bates, F. S.; Macromolecules, 29, 1091(1996)

[20] Khokhlov, A. R.; Kramarenko, E. Y., Macromolecules 29, 681(1996)

[21] Clarke, J. H. R.; Smith, W.; Woodcock, L.V.; J. Chem. Phys. 84,2290 (1986)

[22] Semenov, A. N.; Sov. Phys, JETP, 61, 733(1985)

[23] Helfand, E.; Wassermann, Z.R.; Macromolecules, 9, 879(1976); 11, 960(1978); 13, 994(1980)

[24] Banaszak, M. and Clarke. J. H. R., unpublished data

[25] Banaszak, M. and Clarke. J. H. R,, Phys. Rev. E60, 5753(1999)

[26] Banaszak, M. and Clarke. J. H. R., submitted for publication 